\title{
El ingreso en el hogar y su costo directo en Cuba
}

\author{
Isabel M. Barroso Utra, ${ }^{1}$ Anai García Fariñas, ${ }^{1}$ Armando Rodríguez Salvá, ${ }^{1}$ \\ Pol de Vos, ${ }^{2}$ Mariano Bonet Gorbea ${ }^{1}$ y Patrick Van Der Stuyft ${ }^{2}$
}

Forma de citar Barroso Utra IM, García Fariñas A, Rodríguez Salvá A, de Vos P, Bonet Gorbea M, Van Der Stuyft P. El ingreso en el hogar y su costo directo en Cuba. Rev Panam Salud Publica. 2007;21(2/3): $85-95$.

RESUMEN Objetivos. Analizar el tiempo de estadía en ingreso en el hogar (IH) y su costo diario y por paciente en Cuba, según el diagnóstico al ingreso y la zona donde se brinda el servicio.

Métodos. Se analizó la información de los 837 pacientes en IH entre julio de 2001 y junio de 2002 en un área del municipio Playa, en Ciudad de La Habana (zona urbana metropolitana), una del municipio Cruces, en Cienfuegos (zona urbana no metropolitana), otra del Municipio Unión de Reyes, en Matanzas (zona rural) y una de Fomento, en Sancti Spiritus (zona rural montañosa). Se analizó la mediana del tiempo del IH mediante curvas de supervivencia Kaplan Meier y se evaluó el efecto del diagnóstico al ingreso (afecciones respiratorias, gastrointestinales, ginecobstétricas, egreso hospitalario precoz y otras causas), la zona del $\mathrm{IH}$, el sexo y la edad, sobre la posibilidad de egresar del IH. Para cada municipio se calculó el costo directo del servicio, el costo por paciente y el costo por día de estadía. Se ajustaron modelos de regresión lineal múltiple para identificar el efecto que sobre el costo por paciente tienen el tiempo de estadía, el diagnóstico al ingreso y el área donde se presta el servicio de $\mathrm{IH}$.

Resultados. La causa más frecuente de IH en los municipios estudiados fueron las afecciones respiratorias $(31,4 \%)$, seguidas del egreso hospitalario precoz $(15,5 \%)$, las afecciones ginecobstétricas $(10,8 \%)$ y los trastornos gastrointestinales (8,1\%). La mediana del tiempo de estadia fue de 6 dias (IC95\%: 5,75 a 6,25). En Fomento los pacientes tuvieron 66\% menos probabilidad de egresar que en Cruces y 30\% menos que en Playa y Unión de Reyes. El costo directo del IH en los municipios estudiados medido en pesos cubanos (\$) varió entre \$3 983,54 y $\$ 9624,87$. El costo por día de estadía fue de $\$ 2,57$ a $\$ 6,88$, mientras el costo por paciente fue de $\$ 23,04$ a $\$ 42,78$. El tiempo de estadía tuvo un efecto intrínseco sobre el costo por paciente $(\mathrm{P}<0,0001)$.

Conclusiones. El tiempo de estadía resultó mayor en el área rural montañosa, sin embargo, esta fue el área que generó los menores costos por paciente y los menores costos por día de estadia. El tiempo de estadía puede utilizarse como un indicador de la calidad del servicio del ingreso en el hogar. Desde el punto de vista económico, el tiempo en IH debe analizarse según el diagnóstico al ingreso y el área geográfica.

Palabras clave Servicios de atención de salud a domicilio, tiempo de internación, gastos de hospitalización, Cuba.

\begin{abstract}
Instituto Nacional de Higiene, Epidemiología y Microbiología, División de Epidemiología y Salud Pública, Ciudad de La Habana, Cuba. La correspondencia debe dirigirse a Isabel M. Barroso Utra,
\end{abstract}

Instituto Nacional de Higiene, Epidemiología y Microbiología, Infanta No. 1158 entre Clavel y Llinás, Centro Habana, CP 10300, Ciudad de La Habana, Cuba. Correo electrónico: ibarrosoc@yahoo.es
2 Instituto de Medicina Tropical Príncipe Leopoldo, Departamento de Salud Pública, Unidad de Epidemiología y Control de Enfermedades, Amberes, Bélgica. 
Los servicios de cuidados médicos a domicilio se prestan básicamente en dos modalidades: la hospitalización domiciliaria (HD) y los cuidados de atención primaria de salud a domicilio (APD). La HD surgió en 1947 en Nueva York (1) y se extendió una década más tarde a Europa $(2,3)$ como una alternativa asistencial dirigida a brindar cuidados médicos y de enfermería de nivel hospitalario en los domicilios de los pacientes por parte del propio equipo hospitalario $(4,5)$. Los servicios de APD se pueden prestar según el modelo tradicional o siguiendo el modelo de atención compartida. El primero, que se aplica en la mayoría de los países, no garantiza la continuidad de los servicios ofrecidos en el hospital una vez que pasa a la atención primaria de salud. El segundo, en cambio, permite establecer una alianza estratégica entre los equipos de los dos niveles de atención (5).

En Cuba, los servicios de APD, conocida como ingreso en el hogar (IH), se centran desde 1986 en el médico y el enfermero de la familia (6), que forman el equipo básico de salud (EBS) de la atención primaria y atienden a una población determinada geográficamente. El IH cuenta con la asesoría de los profesores de los grupos básicos de trabajos (GBT) -integrados por especialistas en medicina interna, ginecobstetricia, pediatría y psicologíaradicados en un policlínico local y responsables de atender entre 15 y 20 EBS.

La orden de IH puede basarse en el criterio médico del equipo de atención primaria de salud, el cual evalúa las características de la enfermedad, el estado general del paciente, la situación socioeconómica de la familia, las condiciones higiénicas y sanitarias de la vivienda, las posibilidades de la familia de poder participar en el ingreso y la voluntariedad del paciente (7). Además, la orden de IH puede provenir de los especialistas del nivel secundario de salud, previa coordinación con el equipo de atención primaria de salud $(8,9)$.

El IH tiene carácter transitorio y termina cuando se da de alta al paciente por su mejoría, pasa a régimen de se- guimiento ambulatorio, se refiere a algún servicio hospitalario o muere. Cuando no se presta la atención debida al carácter temporal del $\mathrm{IH}$, los servicios de atención primaria de salud pueden resultar sobrecargados, con el consiguiente perjuicio de las tareas curativas, preventivas y de promoción de salud que tienen a su cargo. El aumento injustificado del tiempo de IH provoca el consumo excesivo de recursos humanos y materiales y reduce la eficiencia del sistema. De esta forma, el tiempo del IH puede utilizarse como un indicador del control de la calidad, similar al tiempo de ingreso hospitalario (10).

En la literatura científica se describe una gran variedad de modelos de aplicación de servicios médicos a domicilio en los que predominan el desconocimiento de los pacientes y la renuencia de los médicos a utilizar esta modalidad de atención primaria (5). Sin embargo, no hay suficientes estudios que analicen su aplicación concreta de acuerdo con el lugar y las características específicas - tanto de los pacientes y de los profesionales participantes como de su organización y de los mecanismos de financiación- en sistemas sanitarios concretos (5).

En el presente artículo se analiza el tiempo de estadía en IH y su costo diario y por paciente según el diagnóstico al ingreso y las características de la zona donde se brinda el servicio. Además, se caracteriza el patrón de comportamiento del IH según los diagnósticos de ingreso más frecuentes en Cuba.

\section{MATERIALES Y MÉTODOS}

Para el presente estudio se analizó la información de los pacientes en régimen de IH entre julio de 2001 y junio de 2002 en cuatro GBT que reflejaran las condiciones de las diferentes áreas geográficas cubanas, según los criterios de la Oficina Nacional de Estadísticas de Cuba.

La muestra quedó conformada por los 837 pacientes en $\mathrm{IH}$ en el área de atención del GBT No. 3 del Policlínico 26 de Julio en el municipio Playa, pro- vincia de Ciudad de La Habana (área urbana metropolitana); del GBT No. 1 del Policlínico Comunitario de Cruces, en el municipio Cruces, provincia de Cienfuegos (área urbana no metropolitana); del GBT del Policlínico Mártires del 13 de Marzo, en los poblados de Cabezas y Bermeja, en el municipio Unión de Reyes, provincia de Matanzas (área rural); y del GBT No. 2 del Policlínico Comunitario de Fomento, en el municipio Fomento, provincia de Sancti Spiritus (área rural montañosa). En lo adelante las áreas estudiadas se identificarán con el nombre del municipio al que pertenecen.

Se diseñó una hoja de datos para recoger la información personal de cada paciente, sus características sociodemográficas, las fechas de ingreso y egreso, el diagnóstico al ingreso y al egreso, la causa de salida del sistema (alta, referencia a algún servicio especializado o muerte) y toda la información relacionada con los recursos humanos y materiales empleados en su atención. Los diagnósticos al ingreso se agruparon según la Clasificación Internacional de Enfermedades, 9. ${ }^{\mathrm{a}}$ revisión (CIE-9) (11) en: afecciones gastrointestinales (códigos: 001-009, 120129, 555-558), respiratorias (códigos: 460-466, 470-478, 480-487, 490-496), ginecobstétricas (códigos: 610, 611, 614-676), cardiovasculares (códigos: 401-405, 410-429), dermatológicas (códigos: 680-709), urinarias (códigos: 580-589) e infecciosas (códigos 052, 057, 061, 100). Se tomó nota, además, si los pacientes tuvieron un egreso hospitalario precoz, definido como el alta hospitalaria de un paciente que, según el criterio de la institución que le dio el alta, requería de cuidados médicos o de enfermería continuos en el hogar, de manera que el paciente egresa del hospital con la indicación de IH. Además, se identificaron los pacientes con afecciones en estadios terminales -es decir, enfermedades que condujeran inexorablemente a la muerte- y que requirieran de cuidados paliativos continuos.

El tiempo de estadía en IH —días que transcurrieron desde la fecha de ingreso hasta la fecha de egreso del paciente- varía considerablemente 
entre pacientes ingresados con diagnósticos similares debido al efecto de las observaciones extremas. Con el propósito de minimizar este efecto se utilizó para el análisis la mediana del tiempo de estadía y su intervalo de confianza de 95\% (IC95\%) por área geográfica y según los diagnósticos al ingreso más frecuentes. Estas estimaciones se obtuvieron mediante curvas de supervivencia por el método del producto límite de Kaplan Meier (12), para lo cual se tomó el tiempo de estadía como variable de respuesta. Se usó la prueba del rango logarítmico (13) para analizar las diferencias entre las curvas de supervivencias. Se ajustaron modelos de regresión proporcional de Cox (14) para evaluar el efecto del diagnóstico al ingreso (afecciones respiratorias, gastrointestinales, ginecobstétricas, egreso hospitalario precoz y otras causas), el lugar del IH (área urbana metropolitana, urbana no metropolitana, rural y rural montañosa), el sexo y la edad, sobre la razón de posibilidades de egresar del IH, expresado como el riesgo relativo (RR) de salir del servicio. Para el análisis estadístico se empleó el programa SAS versión 6.12 (15) y para elaborar las curvas de supervivencias de Kaplan Meier se utilizó el programa SPSS 11.0 (16).

Para cada municipio se calculó el costo directo del servicio, el costo por paciente (costo directo del servicio entre el número de pacientes ingresados) y el costo por día de estadía (costo directo del servicio entre el número de días de estadía), expresados en pesos cubanos del año 2002, según la tasa oficial de cambio establecida por el Ministerio de Finazas y Precios de Cuba (1 peso cubano [\$] = 1 dólar estadounidense [U\$S]).

Para la formación del costo directo se tomaron en cuenta los salarios, el material gastable utilizado, las pruebas diagnósticas realizadas y el tratamiento farmacológico empleado. Para calcular el costo en salario se utilizó el salario por hora tanto del médico y del enfermero de la familia, como de los miembros del GBT y de otros especialistas, según el tiempo dedicado a la atención del paciente. El costo de material gastable se calculó a partir de la cantidad de material utilizado en el paciente (como gasa, algodón, alcohol y agua oxigenada) y de su precio unitario establecido en el registro de productos del almacén del policlínico al que pertenecía cada GBT. El costo de las pruebas diagnósticas se determinó según el número de exámenes indicados al paciente (radiografías, electrocardiogramas, pruebas clínicas y estudios microbiológicos, entre otros) y su precio unitario establecido por el Ministerio de Salud Pública de Cuba (17). Por último, el costo del tratamiento farmacológico se calculó según los medicamentos y los esquemas terapéuticos indicados, el tiempo de duración del tratamiento y el precio de cada medicamento en las farmacias comunita- rias (18). La depreciación de los inmuebles no se tomó en cuenta para el costo directo, ya que el tiempo de uso del consultorio médico de los casos en $\mathrm{IH}$ es mínimo en comparación con el tiempo que el paciente permanece en su casa y no se consideró apropiado utilizar el valor de la vivienda en la que permaneció cada paciente.

Se ajustaron modelos de regresión lineal múltiple para identificar el efecto que sobre el costo por paciente tienen el tiempo de estadía, el diagnóstico al ingreso y el área donde se presta el servicio de IH. Se realizó la transformación logarítmica de los costos por paciente y del tiempo de estadía para linealizar la relación y estabilizar la varianza de los errores (19). Las estimaciones de los costos medios por paciente para hombres y mujeres de edad media se agruparon según el área geográfica de IH y los diagnósticos más frecuentes al ingreso. Dado el número relativamente pequeño de observaciones después de estratificar por área geográfica y diagnóstico, no se presenta un análisis detallado de los costos unitarios.

\section{RESULTADOS}

Durante el año de estudio se informaron 837 casos de IH en las áreas estudiadas de los cuatro municipios seleccionados (cuadro 1). En Fomento se registró la mayor cantidad de $\mathrm{IH}$ por

CUADRO 1. Ingresos en el hogar según el género, el grupo de edad y el grupo básico de trabajo

\begin{tabular}{|c|c|c|c|c|c|c|c|c|c|c|c|c|c|c|c|c|c|c|c|c|c|c|c|c|}
\hline \multirow{3}{*}{$\begin{array}{c}\text { Grupo de } \\
\text { edad, años }\end{array}$} & \multicolumn{6}{|c|}{ Playa } & \multicolumn{6}{|c|}{ Unión de Reyes } & \multicolumn{6}{|c|}{ Cruces } & \multicolumn{6}{|c|}{ Fomento } \\
\hline & \multicolumn{2}{|c|}{ Hombres } & \multicolumn{2}{|c|}{ Mujeres } & \multicolumn{2}{|c|}{ Total } & \multicolumn{2}{|c|}{ Hombres } & \multicolumn{2}{|c|}{ Mujeres } & \multicolumn{2}{|c|}{ Total } & \multicolumn{2}{|c|}{ Hombres } & \multicolumn{2}{|c|}{ Mujeres } & \multicolumn{2}{|c|}{ Total } & \multicolumn{2}{|c|}{ Hombres } & \multicolumn{2}{|c|}{ Mujeres } & \multicolumn{2}{|c|}{ Total } \\
\hline & No. & $\%$ & No. & $\%$ & No. & $\%$ & No. & $\%$ & No. & $\%$ & No. & $\%$ & No. & $\%$ & No. & $\%$ & No. & $\%$ & No. & $\%$ & No. & $\%$ & No. & $\%$ \\
\hline Menos de 5 & 16 & 31,4 & 18 & 21,4 & 34 & 25,2 & 42 & 40,4 & 43 & 35,5 & 85 & 37,8 & 54 & 43,2 & 43 & 26,5 & 97 & 33,8 & 14 & 19,7 & 8 & 6,7 & 22 & 11,6 \\
\hline De 5 a 14 & 10 & 19,6 & 4 & 4,8 & 14 & 10,4 & 14 & 13,5 & 9 & 7,4 & 23 & 10,2 & 6 & 4,8 & 10 & 6,2 & 16 & 5,6 & 10 & 14,1 & 3 & 2,5 & 13 & 6,8 \\
\hline De 15 a 24 & 2 & 3,9 & 5 & 6,0 & 7 & 5,2 & 3 & 2,9 & 8 & 6,6 & 11 & 4,9 & 3 & 2,4 & 13 & 8,0 & 16 & 5,6 & 7 & 9,9 & 23 & 19,3 & 30 & 15,8 \\
\hline De 25 a 49 & 6 & 11,8 & 26 & 31,0 & 32 & 23,7 & 8 & 7,7 & 26 & 21,5 & 34 & 15,1 & 21 & 16,8 & 54 & 33,3 & 75 & 26,1 & 19 & 26,8 & 63 & 52,9 & 82 & 43,2 \\
\hline De 50 a 59 & 5 & 9,8 & 6 & 7,1 & 11 & 8,1 & 5 & 4,8 & 5 & 4,1 & 10 & 4,4 & 7 & 5,6 & 8 & 4,9 & 15 & 5,2 & 6 & 8,5 & 6 & 5,0 & 12 & 6,3 \\
\hline 60 ó más & 12 & 23,5 & 25 & 29,8 & 37 & 27,4 & 32 & 30,8 & 30 & 24,8 & 62 & 27,6 & 34 & 27,2 & 34 & 21,0 & 68 & 23,7 & 15 & 21,1 & 16 & 13,5 & 31 & 16,3 \\
\hline Total & 51 & 37,8 & 84 & 62,2 & 135 & 100 & 104 & 46,2 & 121 & 53,8 & 225 & 100 & 125 & 43,6 & 162 & 56,4 & 287 & 100 & 71 & 37,4 & 119 & 62,6 & 190 & 100 \\
\hline
\end{tabular}


habitantes con 190 y una tasa mensual de 2,9 ingresos por 1000 habitantes. En orden decreciente le siguieron Unión de Reyes (225 ingresos), Cruces (287 ingresos) y Playa (135 ingresos) con tasas mensuales de 2,3, 2,2 y 1,2 ingresos por 1000 habitantes, respectivamente.

El perfil de edad y sexo de las personas que permanecieron en $\mathrm{IH}$ en los diferentes municipios fue muy similar, con la excepción del grupo de mujeres de 25 a 49 años de edad en Fomento, que representaron el $52,9 \%$ de los ingresados en esa área, y los niños menores de 5 años en Unión de Reyes, que constituyeron el 37,8\% de los casos de $\mathrm{IH}$ en ese municipio (cuadro 1).

La causa más frecuente de IH en el conjunto de municipios estudiados fueron las afecciones respiratorias $(31,4 \%)$, seguidas del egreso hospitalario precoz $(15,5 \%)$, las afecciones ginecobstétricas $(10,8 \%)$ y los trastornos gastrointestinales $(8,1 \%)$ (cuadro 2). Las afecciones respiratorias fueron también la causa más frecuente de IH en cada uno de los municipios estudiados. El egreso precoz del hospital fue la segunda causa de ingreso en Cruces $(26,1 \%)$ y Playa $(10,4 \%)$, mientras que en Fomento fueron las afecciones ginecobstétricas $(20,0 \%)$ y en Unión de Reyes las gastrointestinales $(11,6 \%)$ (cuadro 2).

La mediana del tiempo de ingreso para todos los pacientes en IH fue de 6 días (IC95\%: 5,75 a 6,25) y el valor más elevado se observó en las mujeres ingresadas por afecciones ginecobstétricas en Fomento (8 días). Aunque los pacientes ingresados en el hogar por egreso hospitalario precoz permanecieron en promedio 5 días en $\mathrm{IH}$, algunos pacientes permanecieron ingresados más de tres semanas por esta causa. El tiempo de estadía promedio de los pacientes con afecciones respiratorias fue de 6 días, con una distribución similar en los cuatro municipios, excepto en Unión de Reyes donde la estadía más prologada fue de 20 días (cuadro 2).

Al comparar las curvas del tiempo de estadía mediante la prueba del rango logarítmico se encontraron diferencias significativas entre los cua-

CUADRO 2. Prevalencia de los diagnósticos más frecuentes de ingreso en el hogar según el grupo básico de trabajo

\begin{tabular}{|c|c|c|c|c|c|c|c|c|}
\hline \multirow[b]{2}{*}{ Afecciones } & \multirow[b]{2}{*}{ Pacientes } & \multirow[b]{2}{*}{$\%$} & \multirow[b]{2}{*}{ Rango } & \multicolumn{5}{|c|}{ Tiempo de estadía, días } \\
\hline & & & & Mediana & $\begin{array}{l}\text { Percentil } \\
25\end{array}$ & $\begin{array}{l}\text { Percentil } \\
75\end{array}$ & Mínimo & Máximo \\
\hline \multicolumn{9}{|l|}{ Unión de Reyes } \\
\hline Respiratorias & 72 & 32,0 & 1 & 6 & 4 & 7 & 1 & 20 \\
\hline Egreso precoz & 15 & 6,7 & 4 & 7 & 4 & 7 & 3 & 13 \\
\hline Ginecobstétricas & 12 & 5,3 & 7 & 7 & 4,5 & 9 & 2 & 11 \\
\hline Gastrointestinales & 26 & 11,6 & 2 & 4 & 2 & 7 & 1 & 13 \\
\hline Cardiovasculares & 8 & 3,6 & 8 & 6 & 5 & 9,5 & 3 & 12 \\
\hline Dermatológicas & 15 & 6,7 & 5 & 6 & 5 & 7 & 2 & 10 \\
\hline Aparato urinario & 15 & 6,7 & 5 & 7 & 5 & 8 & 4 & 13 \\
\hline Infecciosas & 20 & 8,9 & 3 & 3 & 3 & 4,5 & 1 & 12 \\
\hline Otras & 42 & 18,7 & & 7 & 3 & 8 & 1 & 33 \\
\hline \multicolumn{9}{|l|}{ Cruces } \\
\hline Respiratorias & 88 & 30,7 & 1 & 5 & 3 & 6 & 1 & 11 \\
\hline Egreso precoz & 75 & 26,1 & 2 & 4 & 3 & 6 & 2 & 36 \\
\hline Ginecobstétricas & 26 & 9,1 & 3 & 5 & 4 & 7 & 2 & 14 \\
\hline Gastrointestinales & 25 & 8,7 & 4 & 5 & 3 & 7 & 1 & 16 \\
\hline Cardiovasculares & 12 & 4,2 & 6 & 5 & 3 & 6 & 2 & 7 \\
\hline Dermatológicas & 14 & 4,9 & 5 & 6,5 & 4 & 7 & 2 & 13 \\
\hline Aparato urinario & 7 & 2,4 & 8 & 4 & 3 & 5 & 3 & 6 \\
\hline Infecciosas & 11 & 3,8 & 7 & 4 & 4 & 5 & 3 & 8 \\
\hline Otras & 29 & 10,1 & & 6 & 3 & 7 & 1 & 17 \\
\hline \multicolumn{9}{|l|}{ Fomento } \\
\hline Respiratorias & 50 & 26,3 & 1 & 7 & 6 & 7 & 1 & 15 \\
\hline Egreso precoz & 26 & 13,7 & 3 & 6 & 4 & 8 & 2 & 15 \\
\hline Ginecobstétricas & 38 & 20,0 & 2 & 8 & 6 & 14 & 1 & 82 \\
\hline Gastrointestinales & 5 & 2,6 & 7 & 6 & 4 & 7 & 2 & 15 \\
\hline Cardiovasculares & 22 & 11,6 & 4 & 7 & 5 & 13 & 1 & 32 \\
\hline Dermatológicas & 10 & 5,3 & 6 & 7 & 6 & 8 & 2 & 12 \\
\hline Aparato urinario & 13 & 6,8 & 5 & 7 & 6 & 7 & 4 & 8 \\
\hline Infecciosas & 1 & 0,5 & 8 & 21 & 21 & 21 & 21 & 21 \\
\hline Otras & 25 & 13,2 & & 7 & 6 & 7 & 2 & 97 \\
\hline \multicolumn{9}{|l|}{ Playa } \\
\hline Respiratorias & 53 & 39,3 & 1 & 6 & 4 & 7 & 1 & 14 \\
\hline Egreso precoz & 14 & 10,4 & 2 & 5,5 & 5 & 7 & 4 & 25 \\
\hline Ginecobstétricas & 14 & 10,4 & 2 & 3,5 & 3 & 11 & 1 & 25 \\
\hline Gastrointestinales & 12 & 8,9 & 4 & 4 & 2,5 & 6 & 1 & 14 \\
\hline Cardiovasculares & 8 & 5,9 & 5 & 9,5 & 5 & 12,2 & 1 & 38 \\
\hline Dermatológicas & 4 & 3,0 & 8 & 4 & 3 & 5 & 2 & 6 \\
\hline Aparato urinario & 7 & 5,2 & 7 & 7 & 4 & 10 & 3 & 14 \\
\hline Infecciosas & 8 & 5,9 & 6 & 4 & 2 & 7 & 2 & 19 \\
\hline Otras & 15 & 11,1 & & 6 & 5 & 7 & 3 & 16 \\
\hline \multicolumn{9}{|l|}{ Los cuatro municipios } \\
\hline Respiratorias & 263 & 31,4 & 1 & 6 & 4 & 7 & 1 & 15 \\
\hline Egreso precoz & 130 & 15,5 & 2 & 5 & 3 & 7 & 2 & 36 \\
\hline Ginecobstétricas & 90 & 10,8 & 3 & 7 & 4 & 10 & 1 & 82 \\
\hline Gastrointestinales & 68 & 8,1 & 4 & 5 & 3 & 7 & 1 & 16 \\
\hline Cardiovasculares & 50 & 6,0 & 5 & 6 & 5 & 11 & 1 & 38 \\
\hline Dermatológicas & 43 & 5,1 & 6 & 6 & 4 & 7 & 2 & 13 \\
\hline Aparato urinario & 42 & 5,0 & 7 & 6 & 5 & 7 & 3 & 14 \\
\hline Infecciosas & 40 & 4,8 & 8 & 4 & 3 & 5 & 1 & 21 \\
\hline Otras & 111 & 13,3 & & 6 & 4 & 7 & 1 & 97 \\
\hline
\end{tabular}

tro municipios $(P<0,001)$ (figura 1$)$. Al ajustar el modelo de riesgo proporcional se encontró que la probabilidad de egresar fue menor en Fomento. Así, la probabilidad de los pacientes en IH de egresar en cualquier momento en Cruces fue $66 \%$ (RR = 1,66; IC95\%: 1,35 a 2,03), mayor que la probabilidad de 

(curvas de Kaplan Meier)

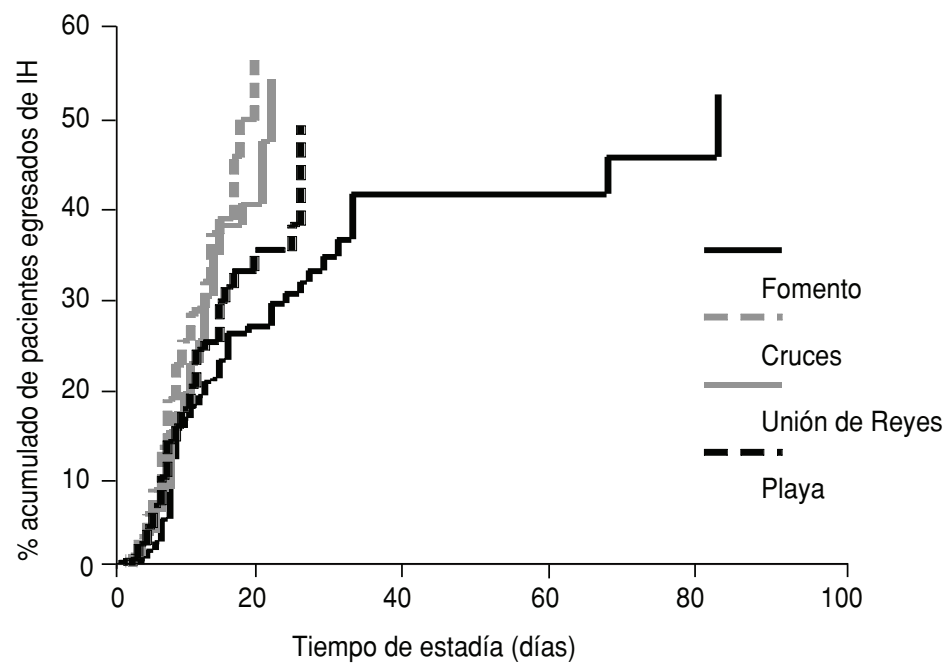

los pacientes en Fomento. Para Unión de Reyes y Playa, esta probabilidad fue mayor aproximadamente en $30 \%$ $(\mathrm{RR}=1,31$; IC95\%: 1,04 a 1,65 y RR = 1,37; IC95\%: 1,11 a 1,69, respectivamente) (cuadro 3).

La probabilidad de egresar cuando la causa de IH eran afecciones ginecobstétricas fue menos de la mitad $(\mathrm{RR}=0,66$; IC95\%: 0,49 a 0,90) que la de los pacientes ingresados por afecciones respiratorias $(P<0,01)$ (cuadro 3). En otras palabras, las mujeres con afecciones ginecobstétricas permanecieron más tiempo en IH que los pacientes ingresados por afecciones respiratorias. Los niños menores de 5 años de edad y los de 5 a 14 años en $\mathrm{IH}$ tuvieron $42 \%$ (RR = 1,42; IC95\%: 1,16 a $1,75)$ y $40 \%$ (RR = 1,40; IC95\%: 1,04 a $1,87)$ mayor probabilidad de egresar, respectivamente, que el grupo de pacientes de 60 años o más (cuadro 3).

Las mujeres con afecciones ginecobstétricas ingresadas en Cruces tuvieron 2,2 veces mayor probabilidad de egresar ( $R R=2,23$; IC95\%: 1,59 a 3,13) que las ingresadas en Fomento, mientras que la probabilidad de egresar de las que se encontraban en IH en Playa fue $56 \%$ mayor $(\mathrm{RR}=1,56$; IC95\%: 1,02 a 2,37) (cuadro 4).

No existen diferencias significativas entre las áreas geográficas o el diagnóstico al ingreso en cuanto al tiempo de $\mathrm{IH}$ de los niños menores de 5 años.

El costo directo del IH de los pacientes en los municipios estudiados varió entre $\$ 3983,54$ y $\$ 9624,87$ (cuadro 5). El costo por día de estadía se encontró entre \$2,57 en Fomento y $\$ 6,88$ en Unión de Reyes. Con la excepción del municipio Playa, a las afecciones en estadio terminal correspondieron los mayores costos por día de ingreso (entre $\$ 6,29$ por día de estadía en Fomento y $\$ 14,86$ en Cruces), seguidas por las afecciones respiratorias. Los menores costos por día de estadía se encontraron en las pacientes con afecciones ginecobstétricas (entre $\$ 2,11$ en Fomento y $\$ 3,80$ en Cruces) (cuadro 5).

Por municipios, el mayor costo por paciente se encontró en Unión de Reyes $(\$ 42,78)$, seguido por Cruces $(\$ 32,33)$, Playa $(\$ 29,51)$ y Fomento $(\$ 23,04)$. Una vez ajustados los efectos que sobre el costo por paciente ejerció el diagnóstico al ingreso y el lugar en que recibió el servicio, se observó que el costo medio por paciente por días de estadía en el IH de los hombres de edad media en Fomento siguió un patrón diferente del resto de los municipios (figura 2), ya que ninguna de las afecciones presentó costos superiores a
$\$ 100,00$ por paciente en este grupo a los 30 días de estadía, a diferencia de las afecciones en estadio terminal en el resto de las áreas geográficas.

En las cuatro GBT estudiadas, las afecciones en estadio terminal tuvieron los mayores costos por paciente, especialmente en Unión de Reyes y Cruces a los 30 días de estadía (\$132,40 y $\$ 126,10$, respectivamente). A diferencia de los restantes municipios, en Fomento no se encontraron diferencias significativas entre los costos por paciente cuando el ingreso fue por afecciones respiratorias, gastrointestinales o por egreso hospitalario precoz.

El patrón de los costos por paciente en las mujeres fue similar al de los hombres, con la excepción de Fomento, donde los costos por paciente con afecciones respiratorias aumentaron de $\$ 43,90$ en los hombres a $\$ 57,10$ en las mujeres a los 30 días de estadía, mayores significativamente que los costos del IH por egreso hospitalario precoz o por afecciones gastrointestinales (figura 3). Además, el costo por paciente de los casos en IH por afecciones ginecobstétricas en las cuatro áreas geográficas fue similar al de los pacientes en IH por egreso hospitalario precoz o por afecciones gastrointestinales. 
CUADRO 3. Razones de posibilidades bruta y ajustada de egresar del hogar

\begin{tabular}{|c|c|c|}
\hline Criterio & $\begin{array}{c}\text { Razón de posibilidades } \\
\text { bruta de egresar y su } \\
\text { IC95\%a }\end{array}$ & $\begin{array}{l}\text { Razón de posibilidades } \\
\text { ajustada de egresar y su } \\
\text { IC95\% }\end{array}$ \\
\hline \multicolumn{3}{|l|}{ Área geográfica } \\
\hline Playa & $\begin{array}{c}1,34 \\
1,07 \text { a } 1,68\end{array}$ & $\begin{array}{c}1,31 \\
1,04 \text { a } 1,65\end{array}$ \\
\hline Unión de Reyes & $\begin{array}{c}1,47 \\
1,20 \text { a } 1,80\end{array}$ & $\begin{array}{c}1,37 \\
1,11 \text { a } 1,69\end{array}$ \\
\hline Cruces & $\begin{array}{c}1,76 \\
1,45 \text { a } 2,12\end{array}$ & $\begin{array}{c}1,66 \\
1,35 \text { a } 2,03\end{array}$ \\
\hline Fomento & Referencia & Referencia \\
\hline \multicolumn{3}{|c|}{ Diagnósticos frecuentes } \\
\hline Gastrointestinales & $\begin{array}{c}1,08 \\
0,83 \text { a } 1,42\end{array}$ & $\begin{array}{c}0,93 \\
0,71 \text { a } 1,23\end{array}$ \\
\hline Ginecobstétricas & $\begin{array}{c}0,61 \\
0,47 \text { a } 0,78\end{array}$ & $\begin{array}{c}0,66 \\
0,49 \text { a } 0,90\end{array}$ \\
\hline Egreso precoz & $\begin{array}{c}0,96 \\
0,78 \text { a } 1,19\end{array}$ & $\begin{array}{c}0.95 \\
0,76 \text { a } 1,19\end{array}$ \\
\hline Otras & $\begin{array}{c}0,89 \\
0,75 \text { a } 1,06\end{array}$ & $\begin{array}{c}0,94 \\
0,79 \text { a } 1,13\end{array}$ \\
\hline Respiratorias & Referencia & Referencia \\
\hline \multicolumn{3}{|l|}{ Género } \\
\hline Hombres & $\begin{array}{c}0,86 \\
0,75 \text { a } 1,00\end{array}$ & $\begin{array}{c}1,00 \\
0,86 \text { a } 1,17\end{array}$ \\
\hline Mujeres & Referencia & Referencia \\
\hline \multicolumn{3}{|l|}{ Grupos de edad, años } \\
\hline Menos de 5 & $\begin{array}{c}1,46 \\
1,20 \text { a } 1,78\end{array}$ & $\begin{array}{c}1,42 \\
1,16 \text { a } 1,75\end{array}$ \\
\hline De 5 a 14 & $\begin{array}{c}1,34 \\
1,01 \text { a } 1,78\end{array}$ & $\begin{array}{c}1,40 \\
1,04 \text { a } 1,87\end{array}$ \\
\hline De 15 a 24 & $\begin{array}{c}0,95 \\
0,71 \text { a } 1,27\end{array}$ & $\begin{array}{c}1,26 \\
0,92 \text { a } 1,73\end{array}$ \\
\hline De 25 a 54 & $\begin{array}{c}0,99 \\
0,81 \text { a } 1,21\end{array}$ & $\begin{array}{c}1,25 \\
1,00 \text { a } 1,56\end{array}$ \\
\hline De 55 a 60 & $\begin{array}{c}1,21 \\
0,87 \text { a } 1,68\end{array}$ & $\begin{array}{c}1,32 \\
0,94 \text { a } 1,83\end{array}$ \\
\hline 60 ó más & Referencia & Referencia \\
\hline
\end{tabular}

a IC95\%: intervalo de confianza de $95 \%$.

CUADRO 4. Razones de posibilidades bruta y ajustada de egresar en mujeres en edad fértil ingresadas en el hogar con diagnóstico al ingreso de afecciones ginecobstétricas

\begin{tabular}{|c|c|c|}
\hline Criterio & $\begin{array}{c}\text { Razón de posibilidades } \\
\text { bruta de egresar y su } \\
\text { IC95\%a }\end{array}$ & $\begin{array}{c}\text { Razón de posibilidades } \\
\text { ajustada de egresar y su } \\
\text { IC95\% }\end{array}$ \\
\hline \multicolumn{3}{|l|}{ Área geográfica } \\
\hline Playa & $\begin{array}{c}1,34 \\
1,01 \text { a } 1,78\end{array}$ & $\begin{array}{c}1,45 \\
0,96 \text { a } 2,20\end{array}$ \\
\hline Unión de Reyes & $\begin{array}{c}1,59 \\
1,22 \text { a } 2,07\end{array}$ & $\begin{array}{c}1,56 \\
1,02 \text { a } 2,37\end{array}$ \\
\hline Cruces & $\begin{array}{c}5,38 \\
4,21 \text { a } 6,88\end{array}$ & $\begin{array}{c}2,23 \\
1,59 \text { a } 3,13\end{array}$ \\
\hline Fomento & Referencia & Referencia \\
\hline \multicolumn{3}{|c|}{ Grupos de edad, años } \\
\hline De 15 a 24 años & $\begin{array}{c}1,05 \\
0,76 \text { a } 1,45\end{array}$ & $\begin{array}{c}1,07 \\
0,77 \text { a } 1,47\end{array}$ \\
\hline De 25 a 49 años & Referencia & Referencia \\
\hline
\end{tabular}

a IC95\%: intervalo de confianza de $95 \%$.

\section{DISCUSIÓN}

Los perfiles por edad y sexo de los pacientes en IH fueron marcadamente diferentes en Unión de Reyes y Fomento. En el primero, se dio preferencia a los niños menores de 5 años, mientras en el segundo predominaron las mujeres entre 25 y 49 años de edad con afecciones ginecobstétricas. Este hecho, además de ser una expresión de la prioridad que en el sistema de salud cubano tiene el programa maternoinfantil, puede estar relacionado con la especialidad de los jefes del servicio de $\mathrm{IH}$ en cada municipio. Por ejemplo, en Unión de Reyes, el médico responsable del servicio de IH era un especialista en pediatría y se ingresaron con mayor frecuencia niños con afecciones más graves que en los municipios restantes. En Fomento, en cambio, la responsable del servicio de IH era una especialista en obstetricia que posiblemente extremaba los cuidados con las gestantes que residían en esa zona rural montañosa, donde es más difícil acceder a los centros médicos. En Fomento se observaron los tiempos de estadía más prolongados.

Este es uno de los primeros trabajos en estudiar el funcionamiento del $\mathrm{IH}$ en Cuba mediante el análisis del tiempo de estadía, como indicador del funcionamiento de este servicio del nivel primario de atención de salud. Este indicador permite analizar el funcionamiento del servicio y la utilización de los recursos, a la vez que posibilita comparar este servicio con el ingreso hospitalario. La metodología empleada permite evaluar servicios similares de atención primaria de salud, sobre todo en países en los que las reformas del sector salud han fomentado el desarrollo del IH.

La HD ha demostrado ser efectiva en función del costo en países europeos y en los Estados Unidos de América (4, $27,31)$ y debe aplicarse en América Latina como una alternativa para fortalecer la atención primaria y de respuesta al crecimiento sostenido de los costos de los servicios de salud. Es necesario desarrollar investigaciones que fundamenten las ventajas de establecer este servicio en estos países y evalúen la ex- 
CUADRO 5. Costos directos (en pesos cubanos) y por días de estadía, según diagnósticos más frecuentes al ingreso y grupo básico de trabajo

\begin{tabular}{|c|c|c|c|c|c|c|c|c|c|c|c|c|}
\hline \multirow[b]{2}{*}{$\begin{array}{c}\text { Afección } \\
\text { diagnosticada } \\
\text { al ingreso }\end{array}$} & \multicolumn{3}{|c|}{ Playa } & \multicolumn{3}{|c|}{ Unión de Reyes } & \multicolumn{3}{|c|}{ Cruces } & \multicolumn{3}{|c|}{ Fomento } \\
\hline & $\begin{array}{l}\text { Costo } \\
\text { directo }\end{array}$ & $\begin{array}{c}\text { Estadía } \\
\text { (días) }\end{array}$ & $\begin{array}{l}\text { Costo por } \\
\text { día de } \\
\text { estadía }\end{array}$ & $\begin{array}{l}\text { Costo } \\
\text { directo }\end{array}$ & $\begin{array}{l}\text { Estadía } \\
\text { (días) }\end{array}$ & $\begin{array}{l}\text { Costo por } \\
\text { día de } \\
\text { estadía }\end{array}$ & $\begin{array}{l}\text { Costo } \\
\text { directo }\end{array}$ & $\begin{array}{l}\text { Estadía } \\
\text { (días) }\end{array}$ & $\begin{array}{l}\text { Costo por } \\
\text { día de } \\
\text { estadía }\end{array}$ & $\begin{array}{l}\text { Costo } \\
\text { directo }\end{array}$ & $\begin{array}{c}\text { Estadía } \\
\text { (días) }\end{array}$ & $\begin{array}{l}\text { Costo por } \\
\text { día de } \\
\text { estadía }\end{array}$ \\
\hline Gastrointestinales & 239,11 & 60 & 3,99 & 571,84 & 127 & 4,50 & 641,19 & 145 & 4,42 & 85,07 & 34 & 2,50 \\
\hline Respiratorias & 1625,01 & 309 & 5,26 & 3757,11 & 447 & 8,41 & 2798,60 & 453 & 6,18 & 1685,53 & 323 & 5,22 \\
\hline Ginecobstétricas & 348,61 & 108 & 3,23 & 282,46 & 79 & 3,58 & 577,22 & 152 & 3,80 & 1120,81 & 532 & 2,11 \\
\hline Egreso precoz & 389,13 & 105 & 3,71 & 543,97 & 100 & 5,44 & 2427,74 & 423 & 5,74 & 674,28 & 171 & 3,94 \\
\hline Estadio terminal & 90,58 & 21 & 4,31 & 1374,57 & 143 & 9,61 & 1144,51 & 77 & 14,86 & 654,56 & 104 & 6,29 \\
\hline Todas las causas & 3983,54 & 891 & 4,47 & 9624,87 & 1399 & 6,88 & 9279,43 & 1553 & 5,98 & 4377,94 & 1704 & 2,57 \\
\hline
\end{tabular}

FIGURA 2. Costos por paciente según el tiempo de estadía en el ingreso en el hogar en hombres de edad media, por municipio y diagnóstico al ingreso
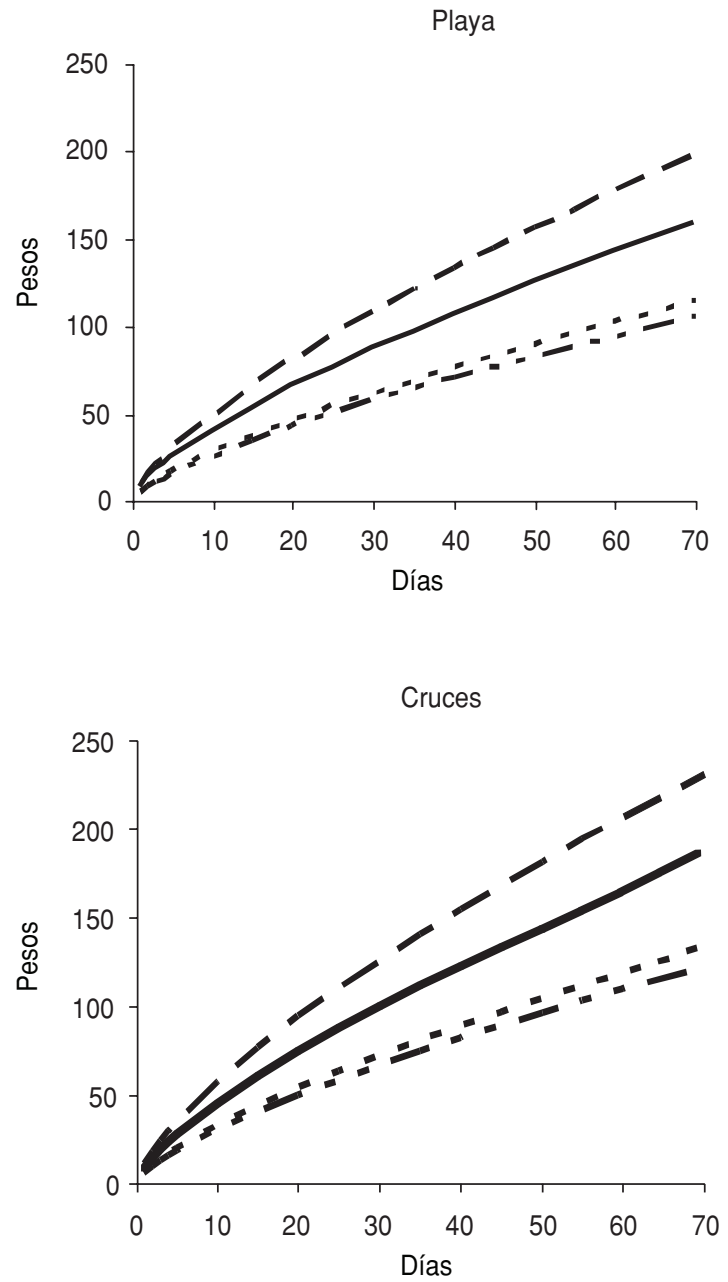

- - Gastrointestinales
- Estadíos terminales

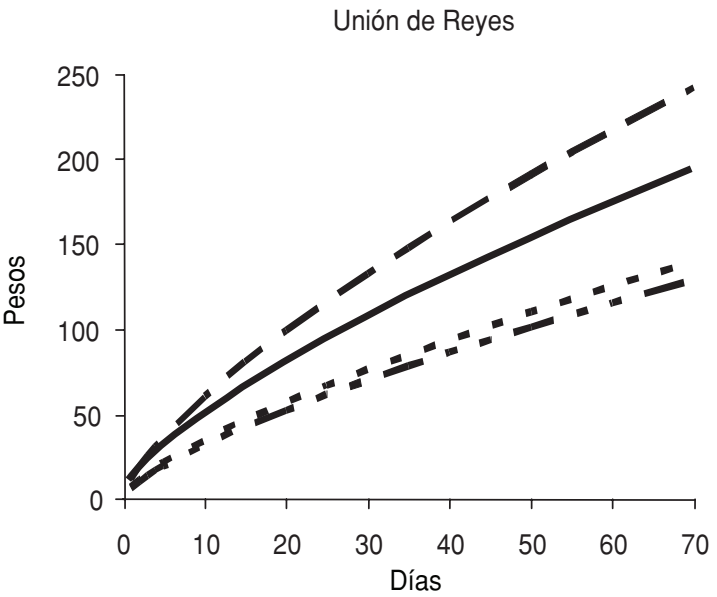

Fomento

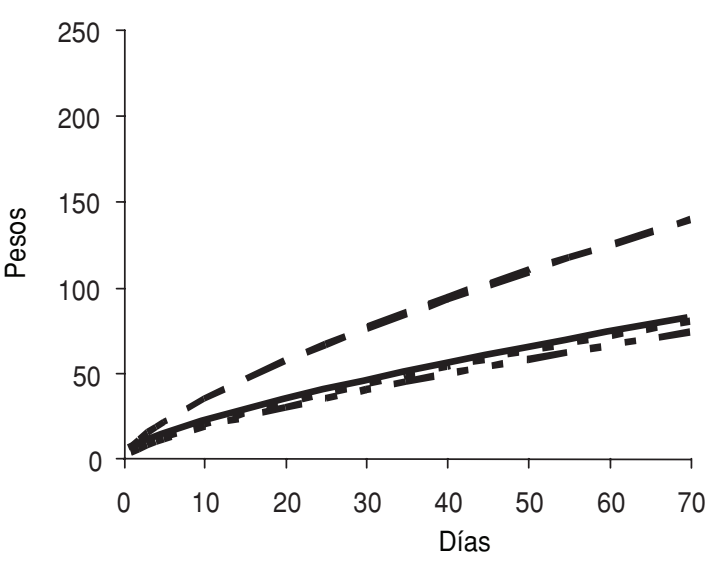

- - - Egreso precoz hospitalario

Respiratorias 
FIGURA 3. Costos por paciente según el tiempo de estadía en el ingreso en el hogar en mujeres de edad media, según el municipio y el diagnóstico al ingreso
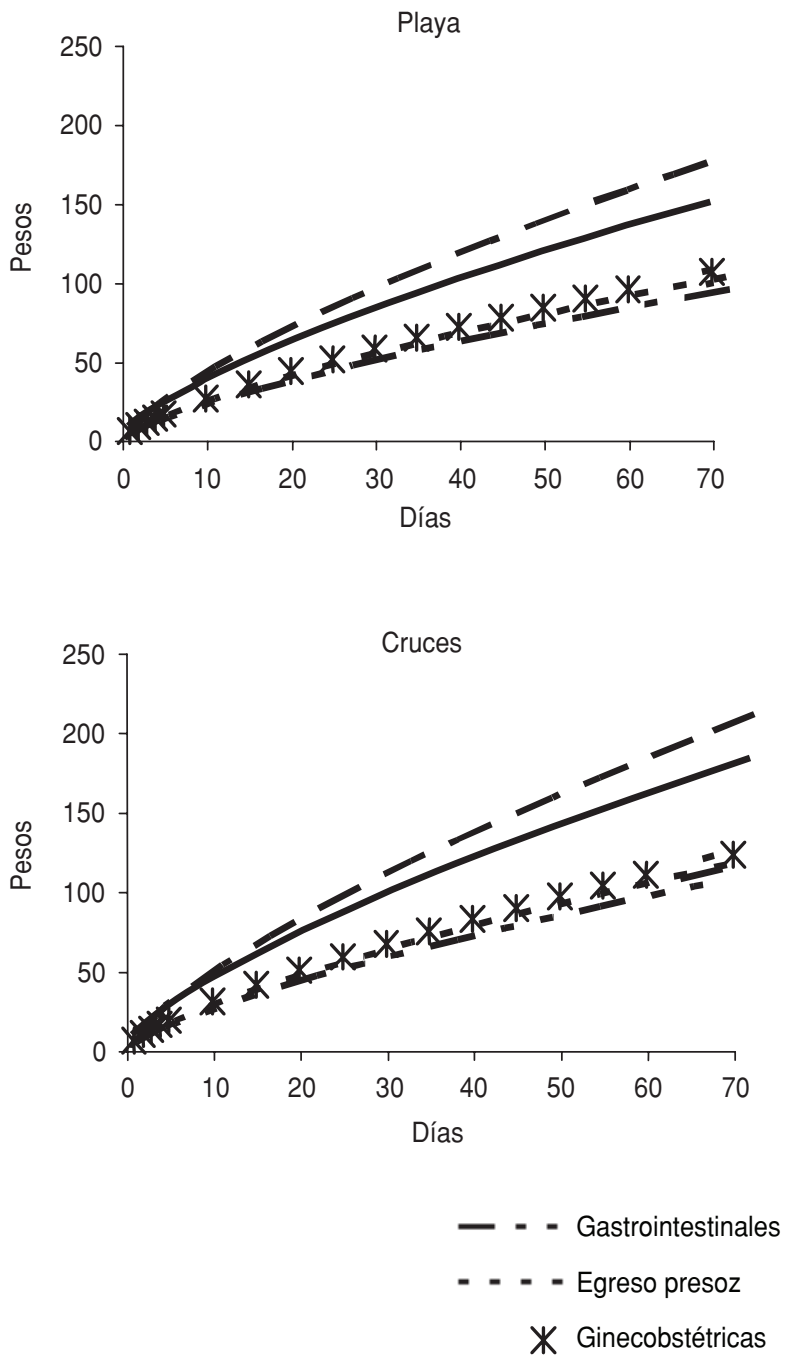
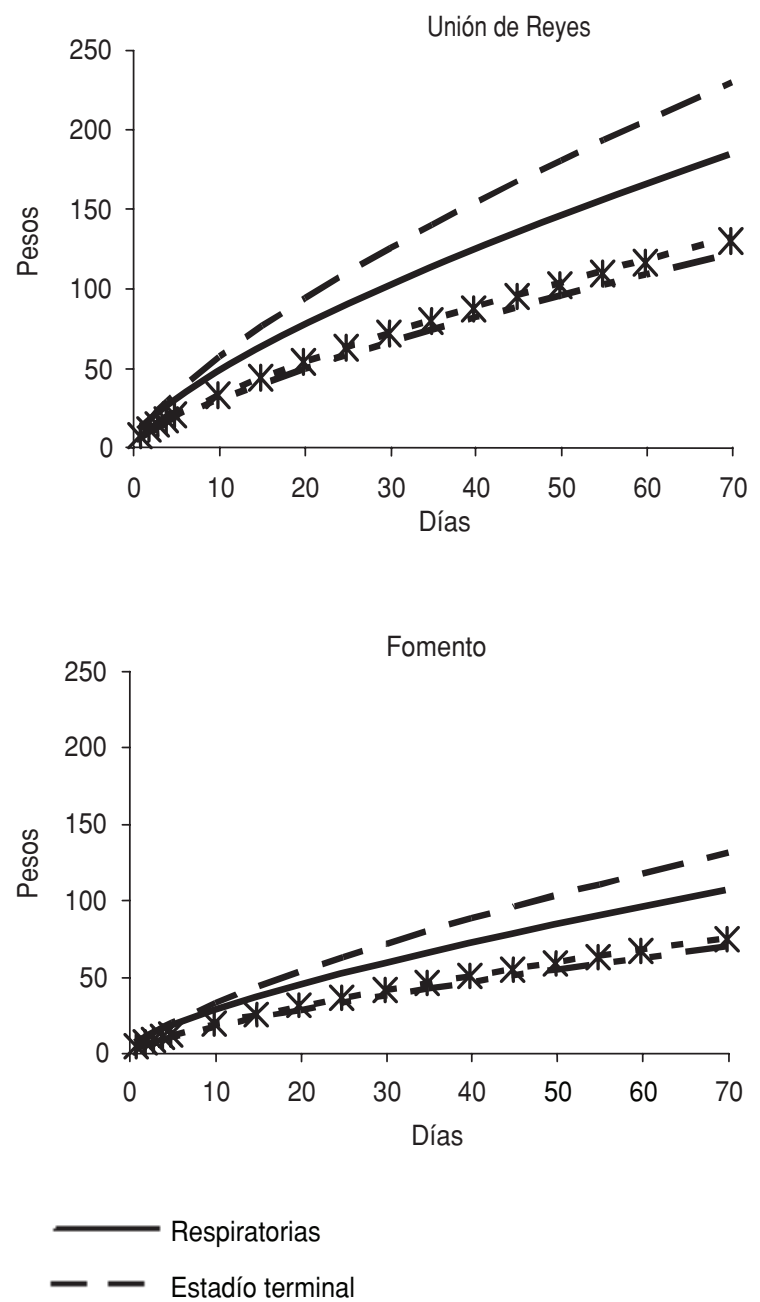

periencia donde se esté aplicando, ya que los resultados pueden variar en dependencia de las características del lugar y de la población.

Aunque en este estudio se utiliza una muestra no aleatoria de GBT, los resultados pueden reflejar el funcionamiento de este servicio en otras áreas similares del país, ya que en Cuba existe un sistema de salud pública único, con cobertura nacional y los GBT cuentan con una estructura organizativa uniforme y se rigen por indicaciones metodológicas nacionales.

El patrón de diagnósticos que motivaron los $\mathrm{IH}$ en los municipios estu- diados fue similar al encontrado por otros autores en Cuba $(20,21)$ y en España (22), con predominio de las afecciones respiratorias como primera causa de ingreso. Sin embargo, aunque los diagnósticos al ingreso son similares, los grupos de edad que hacen uso de este servicio son diferentes, pues en Cuba la mayor proporción de pacientes en IH son menores de 5 años, mientras en España son los mayores de 65 años $(23,24)$.

En el Reino Unido se sigue un patrón diferente, pues los diagnósticos más frecuentes son los trastornos ortopédicos, los ginecológicos y las enfermeda- des en fase terminal (25-27). Aunque, los trastornos ginecológicos constituyen la segunda causa más frecuente de ingreso en el Reino Unido, estos no están relacionados con el embarazo, parto y puerperio como ocurre en Cuba. Por su parte, en Francia, la mayor proporción de pacientes en HD presentan tumores o enfermedades del aparato circulatorio (28). Estas diferencias se deben a que en los países europeos, la HD se destina principalmente a adultos mayores que necesitan cuidados paliativos o atención por enfermedades degenerativas terminales (15, 29). Se debe subrayar que en España, el 
Reino Unido y Francia, la HD aparece como una modalidad diferente a la APD, mientras en los Estados Unidos, la noción de atención domiciliaria integra los conceptos de APD y de HD (30).

El tiempo de estadía informado por diferentes autores varía en dependencia del diagnóstico al ingreso. Según Shepperd (26), los pacientes que se recuperaban de cirugía de rodilla en el Reino Unido tuvieron una media de estadía de 5,72 días; los de cadera, de 6,78 días; las mujeres con histerectomía, de 3,11 días; y los ingresados con enfermedades obstructivas crónicas de las vías respiratorias, de 5,34 días. Coast y colaboradores (31) informaron una estadía media de 10,5 días en pacientes de cirugía en el Reino Unido. En el presente estudio, la mayor proporción de pacientes en IH por egreso hospitalario precoz la constituían pacientes sometidos a cirugía general y la mediana del tiempo de su estadía fue de 5 días, similar a lo informado por Shepperd (26), pero inferior a lo encontrado por Coast y colaboradores (31), aun cuando los diagnósticos más frecuentes en ambos estudios fueron similares.

Fomento fue el municipio con mayores tiempos de estadía. Esto puede deberse al elevado número de $\mathrm{IH}$ por afecciones ginecobstétricas en este municipio y a que estas causan estadías más prolongadas. Además, los pacientes en $\mathrm{IH}$ en este municipio por el resto de las afecciones en general permanecieron mayor cantidad de días ingresados. El hecho de que las pacientes ingresadas por afecciones ginecobstétricas en Fomento fueran las que presentaron tiempos de estadía más prolongados en $\mathrm{IH}$ es un reflejo de las particularidades del funcionamiento del IH en las áreas montañosas. En estas zonas, los pacientes por lo general residen en lugares muy distantes de los centros médicos, tanto de atención primaria como secundaria. Por ello puede entenderse que el IH se convierte en una forma de acercar los servicios de salud a las gestantes con este tipo de afecciones.

En relación con el costo por día de estadía, Caplan y colaboradores encontraron en Australia un costo de 191,00 dólares australianos (U\$S 122,24) por día de servicio en pacientes con enfermedades agudas (32), mientras en el Reino Unido, Wilson y colaboradores calcularon un costo por día de $£ 206,68$ (U\$S 326,55) en adultos mayores con múltiples afecciones (33).

Los costos por paciente calculados en diferentes países varían notablemente. En Bostwana, Moalosi y colaboradores (34) encontraron que el costo de la atención continuada en el hogar de pacientes con tuberculosis crónica fue de U\$S 1 726,00. Li y colaboradores (35) calcularon el costo del tratamiento en el hogar de pacientes con artritis reumatoide en Canadá en 210,87 dólares canadienses (U\$S 141,28). Según Shepperd (26), el costo por paciente de la atención domiciliaria de los adultos mayores con egresos hospitalarios precoces fue de $£ 2$ 279,74 (U\$S 3 442,41), mientras que el de los pacientes con enfermedad crónica obstructiva fue de $£ 2$ 379,7 (U\$S 3 593,35).

En Cuba, Bouza y colaboradores calcularon en $\$ 31,87$ el costo del $\mathrm{IH}$ por paciente, tomando en cuenta solamente el salario del personal asistencial y el costo del tratamiento farmacológico (36), pero esta cifra ascendió a $\$ 58,52$ cuando se contemplaron también los gastos de bolsillo - como alimentación-y de transporte (20). En el presente estudio tampoco se tomaron en cuenta estos gastos.

Aun cuando diferentes autores, tanto cubanos como extranjeros, han estudiado el costo de la HD, es difícil comparar los resultados debido a la diversidad de metodologías empleadas para calcular el costo.

La variación del costo por día de estadía entre las diversas áreas geográficas refleja las diferencias en el empleo de los recursos. Por ejemplo, los mayores costos pueden estar relacionados con el mayor nivel de capacitación de los profesionales de la salud que participan en la atención de los pacientes, con el tiempo que se dedica a visitar a cada paciente o con los tratamientos farmacológicos indicados. Así, los elevados costos por día de estadía observados en los pacientes en IH con enfermedades en estadios terminales están asociados con el incremento del tiempo dedicado a la atención del pa- ciente, mientras el elevado costo por día de estadía de los pacientes con afecciones respiratorias se debe al elevado costo de los medicamentos necesarios y a los esquemas terapéuticos que se indican en estos casos.

El mayor costo por paciente observado en Unión de Reyes puede deberse al ingreso de un número relativamente mayor de pacientes con afecciones respiratorias a los que se les aplicaron esquemas de tratamientos que generalmente no son indicados en la atención primaria y que son más costosos. En el municipio Playa, el costo por paciente fue menor que en los restantes municipios, posiblemente porque el tiempo de estadía promedio fue más breve, los diagnósticos más frecuentes estuvieron balanceados entre enfermedades más costosas - como las enfermedades respiratorias y el egreso hospitalario precoz-y menos costosas - como las afecciones ginecobstétricas y las gastrointestinales-y del ingreso de un número reducido de pacientes en estadio terminal de su enfermedad.

Los diagnósticos más frecuentes - como las afecciones respiratorias, las gastrointestinales y el egreso hospitalario precoz - siguen un patrón de menor estadía en el IH, mientras que las afecciones ginecobstétricas muestran las estadías más largas. Aunque en el área rural montañosa, el tiempo de estadía en IH resultó prolongado, los costos por paciente fueron más bajos que en el resto de las áreas estudiadas y los costos por día de estadía fueron menores. Las restantes áreas tuvieron estadías menores que Fomento y generaron costos por paciente más elevados. En las áreas urbanas, los costos por estadía fueron similares. El área rural (Unión de Reyes) presentó los mayores costos por día de estadía.

Las enfermedades en estadio terminal y las respiratorias fueron las afecciones que generaron los mayores costos por paciente en IH, independientemente de las características de la zona donde se prestó ese servicio. Los costos por paciente originados por las afecciones ginecobstétricas y gastrointestinales, así como por el egreso hos- 
pitalario precoz, fueron bajos y similares en todas las áreas.

Se puede afirmar que el tiempo de estadía puede utilizarse como indicador de la calidad del servicio de IH. Sin embargo, desde el punto de vista económico, el tiempo de estadía debe analizarse según el diagnóstico que da lugar al IH y las características del área geográfica donde se encuentra la unidad de atención que brinda ese servicio.

Agradecimientos. Los autores agradecen a los profesionales que participaron en las labores de campo de los policlínicos 26 de Julio, del municipio
Playa en Ciudad de La Habana; Comunitario de Cruces, del municipio Cruces en la provincia de Cienfuegos; Mártires del 13 de Marzo, en los poblados de Cabezas y Bermeja, en el municipio Unión de Reyes, de Matanzas; y Comunitario de Fomento, del municipio Fomento, en Sancti Spiritus.

\section{REFERENCIAS}

1. Anand JK, Miles JW. Hospital at home, which way will it go? J R Soc Med. 1997;90(7):414-5.

2. Raffy-Pian N. La hospitalización a domicilio: un lugar marginal dentro de los sistemas sanitarios. Rev Gerontol. 1997;7:5-9.

3. González G, Cueto-Felgueroso J, García CA, Menéndez AG. La hospitalización a domicilio: una alternativa a la hospitalización convencional. Medicina Integral. 1995;25(4): 151-5.

4. Shepperd S, Iliffe S. Hospital at home versus in-patient hospital care [Cochrane Review]. En: The Cochrane Library. Issue 1. Chichester, UK: John Wiley \& Sons, Ltd; 2004.

5. Minardi R, Suárez-Varela M, Llopis A, Sette J, Real ER, Días JA. La hospitalización domiciliaria: antecedentes, situación actual y perspectivas. Rev Panam Salud Publica. 2001;10(1): 45-55.

6. Eisen G. La atención primaria en Cuba: el equipo del médico de la familia y el policlínico. Rev Cubana Salud Pública.1996;22(2): 117-24.

7. De la Torre I, Hernández L, Rodríguez ME. Requisitos necesarios para el desarrollo del ingreso en el hogar como forma de atención ambulatoria. Rev Cubana Med Gen Integral. 1998;14(5):479-83.

8. Naranjo J, Delgado A, Forcelledo CR, Camejo MR. Aspectos metodológicos del ingreso en el hogar. Rev Cubana Med Gen Integral. 2000;16(6):606-8.

9. Saura I, Hernández L, Rodríguez ME. Ventajas del ingreso en el hogar como forma de atención ambulatoria. Rev Cubana Med Gen Integral. 1998;14(5):494-8.

10. Lee A, Fung W, Fu B. Analyzing hospital length of stay: mean or median regression? Med Care. 2003;41(5):681-6.

11. Organización Panamericana de la Salud. Clasificación internacional de enfermedades. 9. ${ }^{a}$ rev. Vol I. Washington, D.C.: OPS; 1978. (Publicación científica No. 353).

12. Kapplan G, Meier P. Nonparametric estimation from incomplete observations. J Am Stat Assoc. 1958;53:457-81.

13. Peto R, Peto J. Asymptotically efficient rank invariant test procedures (with discussion). J R Stat Soc A. 1972;135:185-206.
14. Cox DR. Regression models and life-tables. J R Stat Soc (B). 1972;34:187-220.

15. SAS Institute. SAS/STAT User's Guide, Version 8. Cary, NC: SAS Institute Inc.; 1999.

16. SPSS Base 10.0 for Windows. User's Guide. Chicago, IL: SPSS Inc.; 1999.

17. República de Cuba, Ministerio de Salud Pública. Informe nacional de costos, 2002. Ciudad de La Habana: MINSAP; 2002.

18. República de Cuba, Ministerio de Industria Básica. Listado oficial de precios a la población. Cuadro básico, 2002. Ciudad de La Habana: MINBAS; 2002.

19. Box G, Cox DR. An analysis of transformations. J Roy Stat Soc B. 1964;26:211-43.

20. Márquez N, Jiménez L, Torres JH. Costos y beneficios del ingreso en el hogar. Rev Cubana Med Gen Integral. 1998;14(5):445-9.

21. Vera CR, Fernández E, Alonso JL, Basanta L, Zuazaga G. Comportamiento del ingreso en el hogar. Rev Cubana Med Gen Integral. 2001;17(5):429-34.

22. González MDD. Hospitalización a domicilio. Madrid: Hoechst Marion Roussel; 1998.

23. Oterino $D$, Peiró $S$, Marchán $C$, Ridao $M$. Cuidados de enfermería en hospitalización a domicilio y hospitalización convencional. Rev Esp Salud Publica. 1998;72:517-27.

24. Revilla L, Espinosa JM. La atención domiciliaria y la atención familiar en el abordaje de las enfermedades crónicas de los mayores. Aten Primaria. 2003;31(9):587-91.

25. Richards SH, Coast J, Gunnell DJ, Peters TJ, Pounsford J, Darlow MA. Randomised controlled trial comparing effectiveness and acceptability of an early discharge, hospital at home scheme with acute hospital care. $\mathrm{Br}$ Med J. 1998;316:1796-801.

26. Shepperd S, Harwood D, Jenkinson C, Gray A, Vessey M, Morgan P. Randomised controlled trial comparing hospital at home care with inpatient hospital care. I: Three month follow up of health outcomes. Br Med J. 1998;316:1786-91

27. Shepperd S, Harwood D, Gray A, Vessey M, Morgan P. Randomised controlled trial comparing hospital at home care with inpatient hospital care. II: Cost minimisation analysis. Br Med J. 1998;316:1791-6.
28. Shepperd S, Ilife S. Effectiveness of hospital at home compared to in-patient hospital care. En: Bero L, Grilli R, Grimshaw J, Osman A, eds. Collaboration on effective professional practice module of the Cochrane Database of Systematic Reviews. The Cochrane collaboration. Issue 1. Oxford: Updated Software; 1998.

29. Coleman B. European models of long-term care in the home and community. Int J Health Serv. 1995;25:455-74.

30. Brodsky J, Habib J. New developments and issues in home care policies. Disabil Rehabil. 1997;19(14):150-4.

31. Coast J, Richards SH, Peters TJ, Gunnell DJ, Darlow MA, Pounsford J. Hospital at home or acute hospital care? A cost minimisation analysis. Br Med J. 1998;316:1802-6.

32. Caplan GA, Ward JA, Brennan NJ, Coconis J, Board N, Brown A. Hospital in the home: a randomised controlled trial. Med J Aust. 1999;170:156-60.

33. Wilson A, Parker H, Wynn A. Randomised controlled trial of effectiveness of Leicester hospital at home scheme compared with hospital care. Br Med J. 1999;319:1542-6.

34. Moalosi G, Floyd K, Phatshwane J, Moeti T, Binkin N, Kenyon T. Cost-effectiveness of home-based care versus hospital care for chronically ill tuberculosis patients, Francistown, Botswana. Int J Tuberc Lung Dis. 2003;7(Suppl1):S80-5.

35. Li LC, Coyte PC, Lineker SC, Wood H, Renahan M. Ambulatory care or home-based treatment? An economic evaluation of two physiotherapy delivery options for people with rheumatoid arthritis. Arthritis Care Res. 2000;13(4):183-90.

36. Bouza HP, Márquez PM, Martín J, Montano G, Romero RE, Vergel M. Costos, beneficios y satisfacción poblacional del ingreso domiciliario. Medicentro. 2004;8(1).

Manuscrito recibido el 23 de agosto de 2004. Aceptado para publicación, tras revisión, el 31 de enero de 2005. 
ABSTRACT Objective. To analyze home care services in Cuba and determine how length of stay, per-day cost, and per-patient cost vary by diagnosis and by the area of the country in which the services are rendered.

The direct costs of home care in Cuba
Methods. Patient information was analyzed for 837 individuals who were enrolled in home care services between July 2001 and June 2002 in the following four municipalities: (1) Playa municipality (a metropolitan urban area) in the province of the City of Havana; Cruces municipality (an urban, but not metropolitan, area) in the province of Cienfuegos; Unión de Reyes municipality (a rural area) in the province of Matanzas; and Fomento municipality (a mountainous rural area) in the province of Sancti Spiritus Analysis of the mean length of stay for home care services was conducted using the Kaplan-Meier survival curve method. The impact of the following criteria on the probability and timing of discharge was also assessed: diagnosis at time of enrollment (respiratory, gastrointestinal, obstetrical/gynecological, hospital discharge follow-up, and other causes), area in which services were rendered, and patient gender and age. The total service, per-patient, and per-day costs were determined for each municipality. Adjusted multilinear regression models were used to determine how length of stay, diagnosis upon enrollment, and service area affected cost.

Results. The diagnoses most frequently requiring home care were respiratory illness $(31.4 \%)$, hospital follow-up of acute condition (15.5\%), obstetrical / gynecological illness $(10.8 \%)$, and gastrointestinal disorder $(8.1 \%)$. The mean length of stay was 6 days (95\% confidence interval: 5.75 to 6.25$)$. In Fomento, the probability of patients enrolling in home care was 66\% lower than in Cruces and 30\% lower than in Playa and Unión de Reyes. The total direct cost of home care in the municipalities studied ranged, in Cuban pesos, from \$3 983.54 to $\$ 9624.87$. The per-day cost ranged from $\$ 2.57$ to $\$ 6.88$, and the per-patient cost from $\$ 23.04$ to $\$ 42.78$. The length of stay had a direct impact on per-patient cost $(P<0.0001)$.

Conclusions. A longer length of stay was observed in the mountainous rural area; however, this was where the lowest per-patient and per-day costs were incurred. Length of stay can be used as an indicator for measuring the quality of home care services. From a cost perspective, length of stay must be evaluated based on diagnosis and geographic area.

Key words Hospital-based home care services, length of stay, health expenditures, Cuba. 